\title{
A Combined Electron Microscopic-Microangiographic Methodology for Cerebrovascular Studies
}

\author{
Pentti T. Jokelainen, David R. Webb, and Elliott D. Yush \\ University of Michigan, Department of Anatomy, Medical Science II, Ann Arbor, Michigan 48109
}

Received July 27, 1979, and in revised form, December 10, 1979

\begin{abstract}
A combination methodology is introduced which permits sequential observation of the same tissue by microangiographic and electron microscopic techniques. Three-dimensional vascular patterns can be elucidated with specific vascular features of interest accurately identified and isolated for detailed electron microscopic analysis. Perfusion with a fixative providing good ultrastructural preservation is followed by a short vascular flush and subsequent perfusion with radiographic contrast medium in aqueous gelatin solution. Gelling of the medium, effected by reentry of aldehyde fixatives into vessel lumina, retains it in vessels both during subsequent slicing for microangiography and processing for electron microscopy. Specific vascular features are identifiable by gross or light microscopic examination of the high-resolution microangiographic plates and the corresponding areas removed from the Epon-embedded slices for observation by electron microscopy. The differential filling of the vascular bed permits discrimination of arterial from venous segments.
\end{abstract}

In electron microscopic studies of cerebral vasculature and other three-dimensional vascular beds, it is difficult both to locate specific features of infrequent occurrence and to maintain a connection between ultrastructural findings and the three-dimensional context in which they occur. In recent years radiological angiography has been of considerable use in the delineation of angioarchitectural relationships, particularly in cerebral vascular studies. Providing good spatial resolution of the vascular network, it has also been invaluable in the localization and quantitation of focally occurring phenomena. In this report we describe a combination of microangiographic and electron microscopic techniques that permits the initial scanning of entire vascular beds and the identification and isolation for ultrastructural observation of vascular features within their threedimensional associations. This technique additionally facilitates the dependable differentiation of arterial from venous circulations. In the present investigation, this combined methodology was applied to studies of the cerebral vasculature of nor- mal and stroke-prone rats and specifically to the occurrence of focal vascular narrowings.

\section{MATERIALS AND METHODS}

Fixation. Normal male and female Wistar-Kyoto and spontaneously hypertensive stroke-prone rats (13) of about $300 \mathrm{~g}$ average body wt were anesthetized with $10 \mathrm{mg} / \mathrm{kg}$ ketamine, im, followed by 400 USP units $/ \mathrm{kg}$ heparin via a tail vein. Thoracotomy was accomplished by lateral and diaphragmatic incision, leaving the internal thoracic arteries intact for later monitoring of radiographic contrast medium infusion. Cannulation was performed by insertion of a 19-gauge needle through the left ventricle into the ascending aorta. The right atrium was immediately cut, the descending aorta clamped, and perfusion initiated.

The perfusion apparatus $(5,19)$ consisted of three bottles at $140 \mathrm{~cm}$ height connected via $\mathrm{Y}$-type venostat infusion tubing (Abbot Laboratories) and three-way stopcock to a common cannula. The following solutions were freshly made, filtered ( $3 \mu \mathrm{m}$ Millipore), and administered at $38^{\circ} \mathrm{C}$.

(A) Flush solution: $8 \%(\mathrm{w} / \mathrm{v})$ sucrose in $0.1 \mathrm{M}$ cacodylate buffer, $\mathrm{pH} 7.35$.

(B) Weak fixative: $1.0 \%$ glutaraldehyde, $1.25 \%$ paraformaldehyde, $0.09 \% \mathrm{CaCl}_{2}$, in $0.1 \mathrm{M}$ cacodylate buffer, $\mathrm{pH} 7.35,530 \pm 10$ mosm.

(C) Strong fixative: $4 \%$ glutaraldehyde, $5 \%$ paraformaldehyde, $0.09 \% \mathrm{CaCl}_{2}$, in $0.1 \mathrm{M}$ cacodylate buffer, $\mathrm{pH} 7.35,953 \pm 10$ mosm. 
Osmolarities were determined by freezing-point depression, using an osmometer (Advanced Instruments, Inc. Model 31LA). Osmolarity of the buffer in all the above solutions measured $330 \pm 10$ mosm.

The cerebral vascular system was initially perfused with the flush solution (A) for $20 \mathrm{sec}$, followed by fixative solutions (B) and (C) for 5 and $20 \mathrm{~min}$, respectively. Maximal initial flow rates of $20 \mathrm{ml} / \mathrm{min}$ and perfusion pressures of $100 \mathrm{~mm} \mathrm{Hg}$, continuously monitored at the cannula, were employed in all cases. The flow rate was reduced after the observed stiffening of the animal's neck, occurring within the first minutes of fixation.

Microangiography. Following fixative perfusion the vascular bed was rinsed with $200 \mathrm{ml}$ of flush solution (7-10 $\mathrm{min}$ ) to empty the vessels of aldehydes prior to infusion of the radiographic contrast medium, consisting of $25 \%(\mathrm{v} / \mathrm{v})$ Micropaque (barium sulfate dispersion, Nicholas Laboratories Ltd., Slough, England) and $2 \%(\mathrm{w} / \mathrm{v})$ gelatin. A Harvard pump was used to infuse $1-2 \mathrm{ml}$ of the medium, $\left(40-42^{\circ} \mathrm{C}\right)$ at $1.5 \mathrm{ml}$ / min, and at pressures never exceeding $130 \mathrm{~mm} \mathrm{Hg}$.

Direct observation of the progressive filling of the internal thoracic artery and its intercostal branches allowed good estimation of the extent of cerebrovascular filling. Selective cerebral arteriolar filling was regularly achieved when the perfusion was interrupted after about $5 \mathrm{sec}$ following the appearance of the opaque mass in the small intercostal arterial branches. Filling of the cerebral venular bed necessitated substantially longer perfusion times and/or higher pressures. Approximately 20 min was allowed for gelling of the contrast medium by gradual rediffusion of aldehydes from parenchyma to the vessels. The brain was carefully removed for gross angiography (Fig. 1), and then serially cut, generally in the coronal plane, to 1mm slices. Each slice was marked with metal pins of known diameter to indicate laterality or the occurrence in the stroke animals of lesions evident at the brain surface, and for calibration of microangiographs. After storage in solution (C) overnight, tissue slices were wrapped in thin polyethylene film to prevent drying during microangiography. Radiographs (Fig. 2) were taken on Kodak Spectroscopic Plates using a Westenskow microradiograph machine (Garth Westenskow Co., Salt Lake City, Utah) at $11 \mathrm{kV}, 20 \mathrm{~mA}$, 3 min. Microangiographs can also be taken on Kodak high-resolution plates using a Faxitron apparatus (6).

Selective electron microscopy. The tissue slices were postfixed with osmic acid, stained en bloc with uranyl acetate, and flat embedded in Epon. Rapid scanning of microangiographs of the 16-20 slices per brain allowed particular vascular segments of interest to be identified both with respect to position in the brain as a whole and within individual slices. The extremely fine grain of the spectroscopic plates (Figs. 4 ,9) enabled microangiographic details to be further resolved by their light microscopic examination. Specific sites thus localized were removed from the corresponding epon embedded slices and sectioned for electron microscopy. Semi-thin and thin sectioning of the barium sulfate infused vessels did not result in dulling of glass or diamond knives. Thin sections were stained with uranyl acetate and lead citrate and examined with a Siemens 101 electron microscope.

\section{RESULTS}

Figures 3-6 represent successive levels of magnification of the same cerebral cortical region examined by the described combination of radiography (Fig. 3), light microscopy of a microangiograph (Fig. 4), and electron microscopy (Figs. 5 and 6). Samples selected from both cortical and medullary regions showed clear radiographic delineation of vascular patterns (Figs. 2, 3,

FIG. 1. Angiograph of normal rat brain which has been fixed for subsequent electron microscopy, delineating major arterial branching patterns. A, M, P, I, anterior, middle, posterior, and inferior cerebral arteries, respectively; $\mathrm{B}$, basilar artery. $\times 5$.

FIG. 2. Microangiograph of a 1-mm-thick coronal slice of the parietal region of the brain taken prior to embedding in epoxy resin. Note the uniform filling of cortical and medullary arterioles. LV, lateral ventricle; $\mathrm{FV}$, fourth ventricle; $\mathrm{AC}$, anterior cerebral artery. $\times 8.4$.

Frgs. 3-6. Microangiographic (Figs. 3 and 4) and electron microscopic (Figs. 5 and 6) representations of the same cortical penetrating arterioles.

FIG. 3. Low-magnification microangiograph used to identify intraparenchymal vessels of interest for electron microscopic sectioning (boxed cortical area $=1.5 \mathrm{~mm}^{2}$ ). $\times 14.5$.

FIG. 4. The boxed area of Fig. 3 as observed by light microscopy of the high-resolution microangiographic plate, showing greater detail of penetrating arterioles branching from the surface pial vessels. Branching point constrictions (arrows) are associated with valvular structures similar to that seen in Fig. $8 . \times 72$.

FIG. 5. Low-magnification electron micrograph of a portion of the penetrating arteriole identified in Fig. 4 $(*)$. Note the presence of barium sulfate granules in the vessel lumen, confirming arteriolar identity. Surrounding cortical parenchyma shows good preservation. NU, nerve cell nucleus. $\times 2000$.

FIG. 6. Detail of the arteriole represented in Fig. 5. Preservation of smooth muscle (SM) and endothelial (EN) components has been unaffected by perfusion with the radiographic contrast medium. LU, vessel lumen. $\times 17500$. 


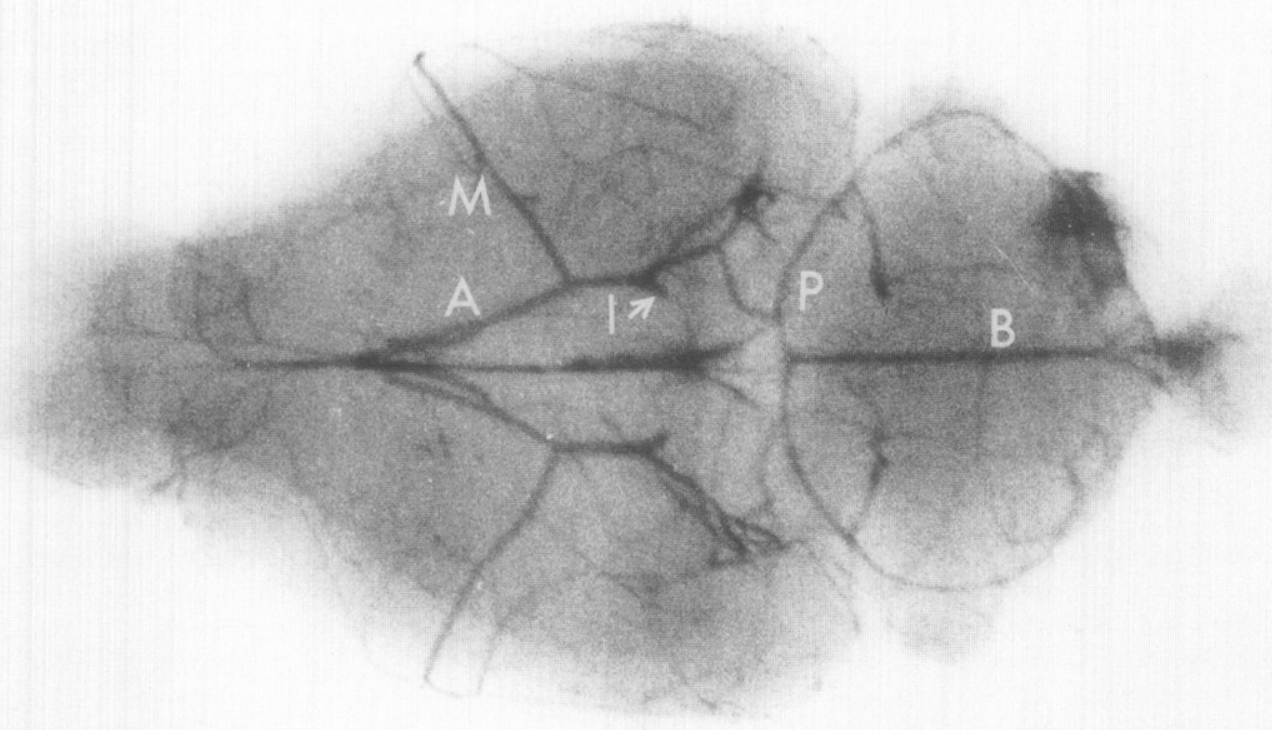

(1)

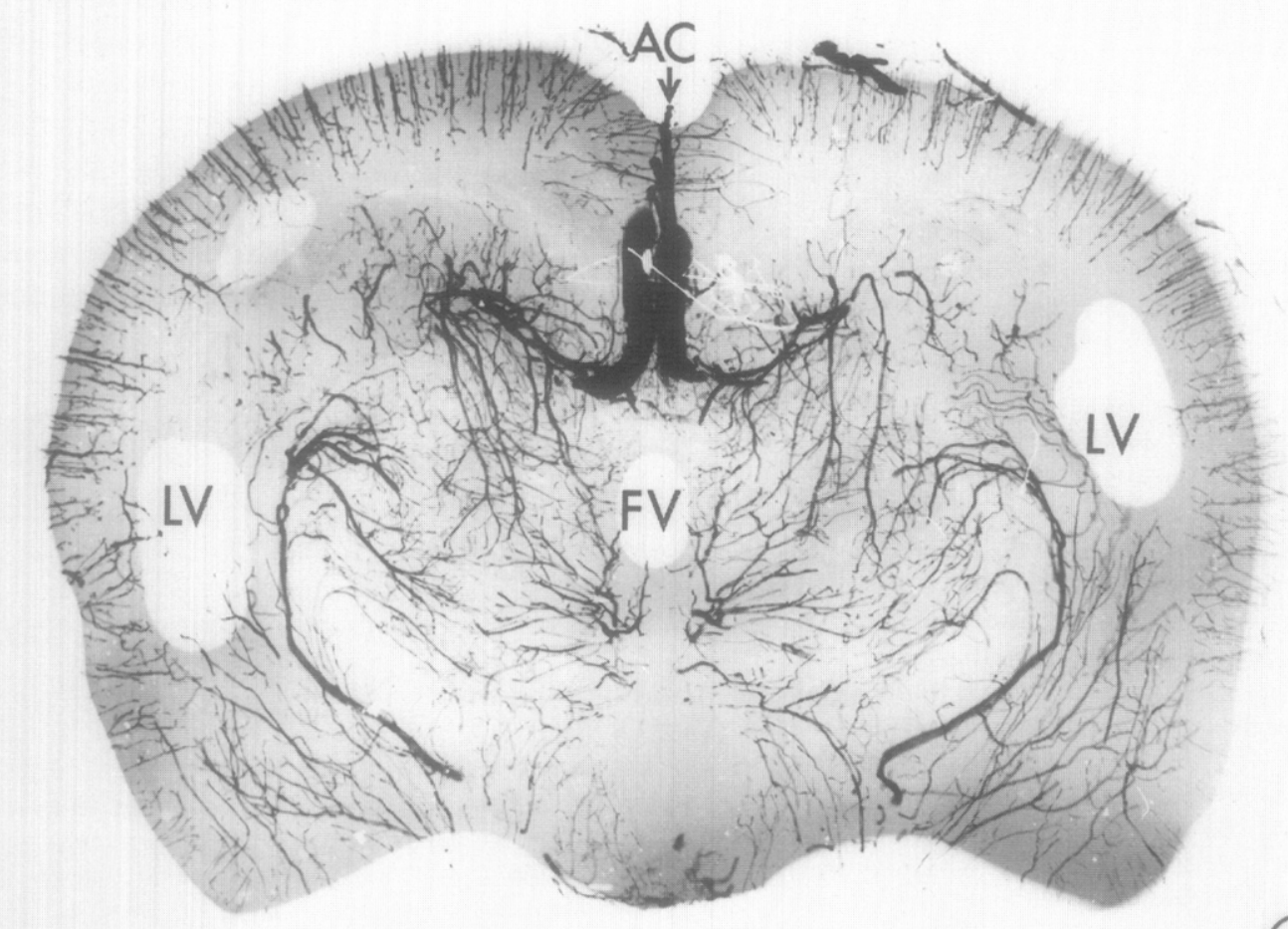



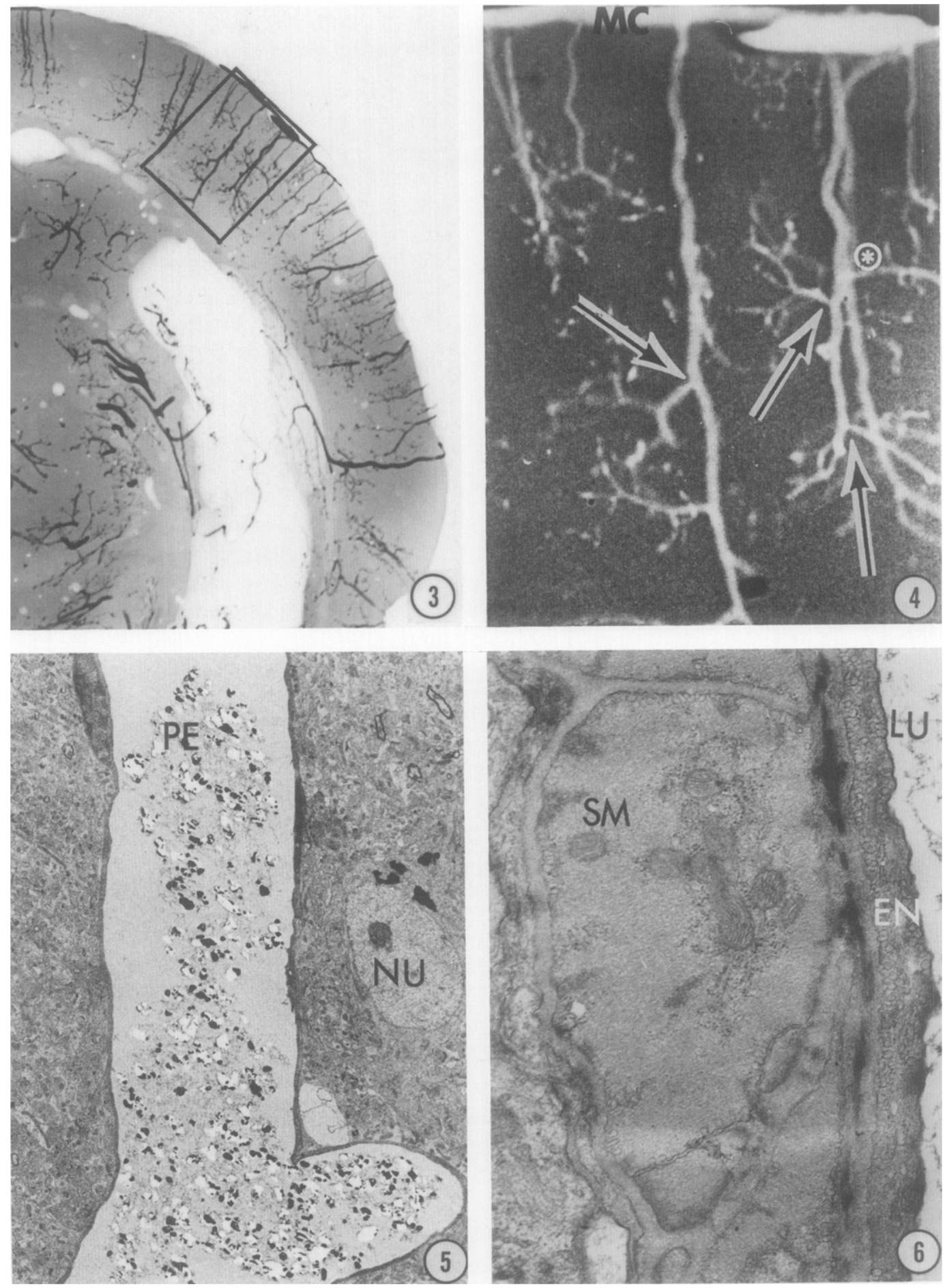
4, and 9), with good ultrastructural preservation of microvascular, glial, and neural components (Figs. 6 and 7). Gross visual examination of the pial vasculature indicated only arterial segments to be filled with contrast material, the empty venous segments appearing as dark profiles (Fig. 8). Whole brain angiography similarly demonstrated filling of the arterial vasculature but not of veins or venous sinuses (Fig. 1). The arterially restricted filling is particularly well evidenced by microangiographs of oblique cortical sections (Fig. 9), demonstrating a continuity of filling from parent pial arterioles through cortical penetrating branches and extending into, but not past, the capillary bed. Angiographic screening of normal rat brain tissues revealed the frequent occurrence of lumenal constrictions at branching points (Figs. 4 and 9) of the superficial cerebral and the intracerebral arterioles down to $15-\mu \mathrm{m}$-diameter branches. Electron microscopy showed these constrictions to be invariably associated with valve-like specializations of the vascular wall (Fig. 10). Similar structures were found to occur in the strokeprone animals but with an apparent degeneration of cellular components. In addition to the branching point constrictions, angiographs of the stroke-prone vasculature demonstrated focal luminal narrowings along the length of a number of cortical arterial vessels (Fig. 11). By electron microscopy these were seen to involve invagination of the arterial wall associated with apparent perivascular edema (Fig. 12).

\section{DISCUSSION}

\section{Preliminaries to Fixation}

Successful combination of the two methodologies requires the total emptying of the vasculature of cellular and plasma components. Preheparinization of the animal resulted in more complete angiographic filling and, when used with a subsequent prefixation vascular flush, produced considerably improved delineation of microvascular patterns of the entire brain. The advisability of prefixation flush has been questioned because of possible resulting ischemic or hypoxic lesions (14). One approach to this potential problem has been to oxygenate the rinsing solution (12).

However, the occurrence of minute gas emboli, a factor responsible for abnormal angiographic filling of vessels (8), would make such an approach particularly unsuitable for microangiographic studies. Tracheotomy and mechanical respiration have been commonly employed to prevent anoxic changes during preliminary surgical procedures $(14,19)$. While debate exists as to the time required for onset of anoxic ultrastructural changes $(4,7,9)$, our use of short operation and flush times (30-40 sec from opening of thorax to inititation of fixation) resulted in excellent preservation of vascular wall and neural parenchyma (Figs. 6 and 7). Lesions commonly associated with anoxia such as glial swelling or disruption of neuronal organelle membranes were rare and of only focal occurrence.

FIG. 7. Electron micrograph of cortical neuropil surrounding small barium sulfate infused vessel, and illustrating good preservation of neural (NE) and associated glial (GL) components. $\times 4300$.

FrG. 8. Photograph of flat Epon-embedded pia showing the selective filling of arteriolar vessels (white profiles) by the radiographic medium. The nonfilled veins can be seen as the dark profiles (arrows) in the background. Light and electron microscopic examination of these veins invariably show empty lumina as contrasted to the barium sulfate-filled arterioles. $\times 35$.

FIG. 9. Microangiograph of a slice from parietal cortex, showing the continuity of branches (MC) of the middle cerebral artery to cortical penetrating arterioles (PE). Venous vessels are not evident. Constrictions (arrows) are seen at the branching sites from the larger pial arterioles. $\times 30$.

Fig. 10. Valve-like structure (arrows) at the branching site of a pial arteriole, appearing as a constriction in the microangiograph of Fig. $9 . \times 2200$. 

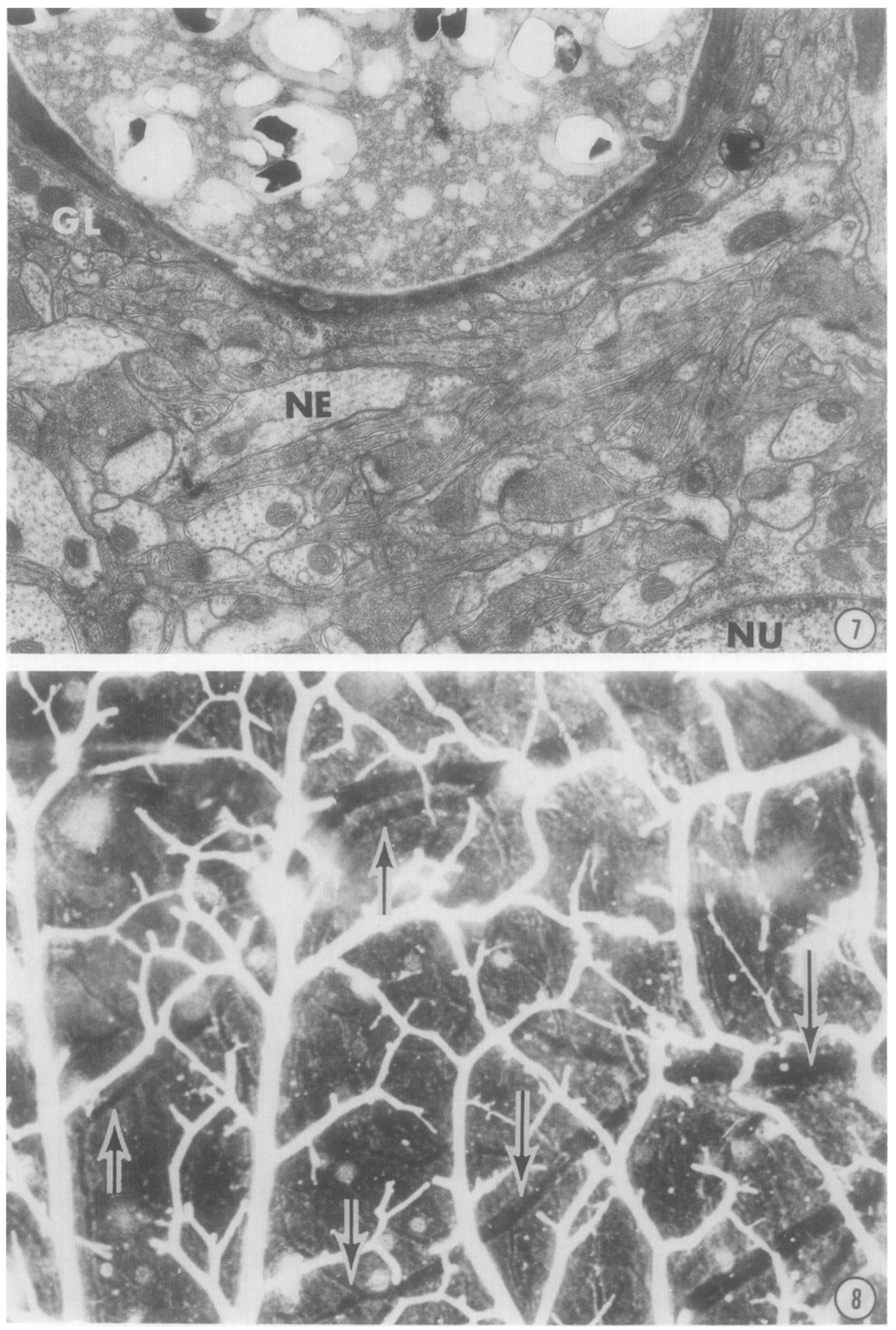

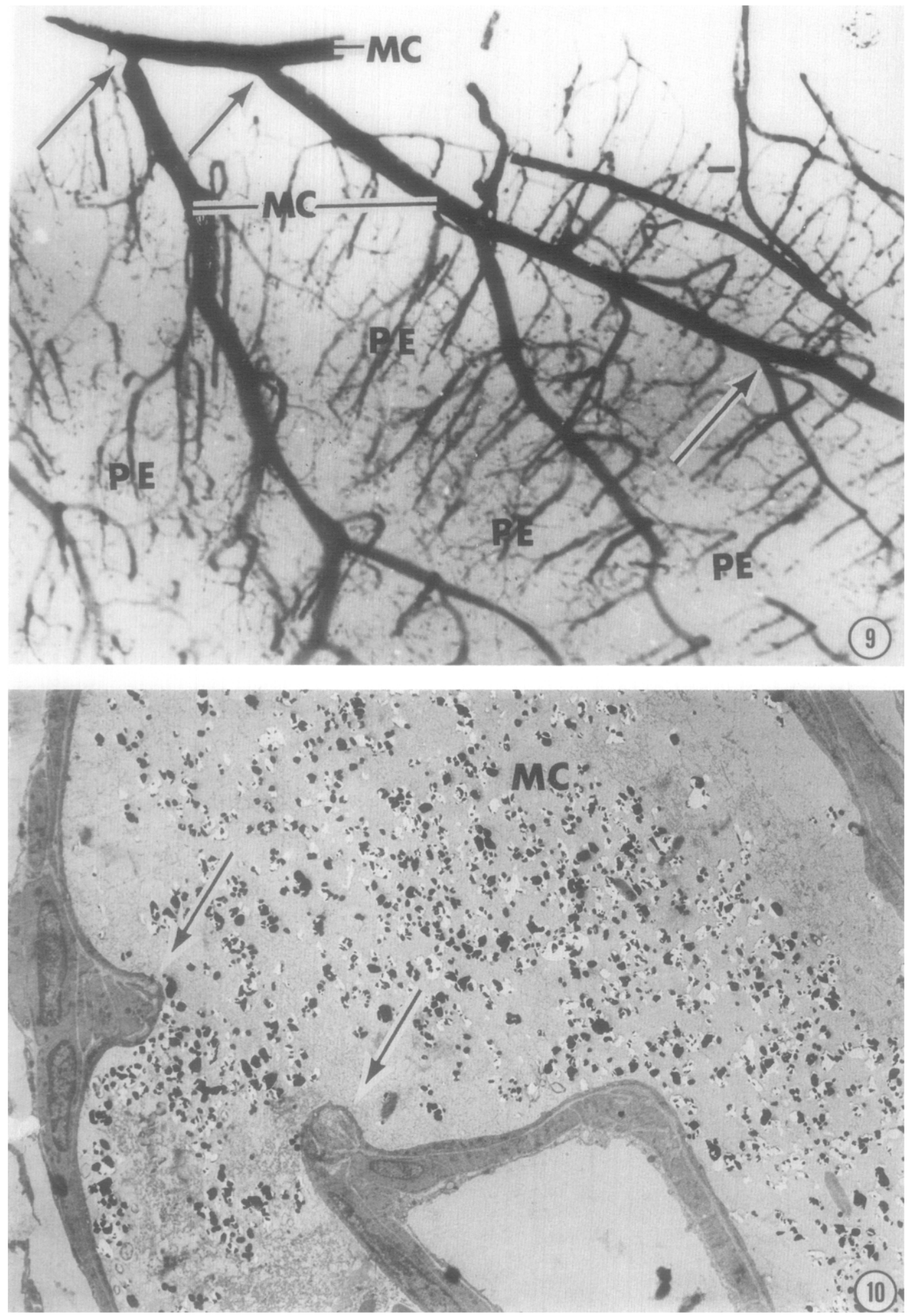

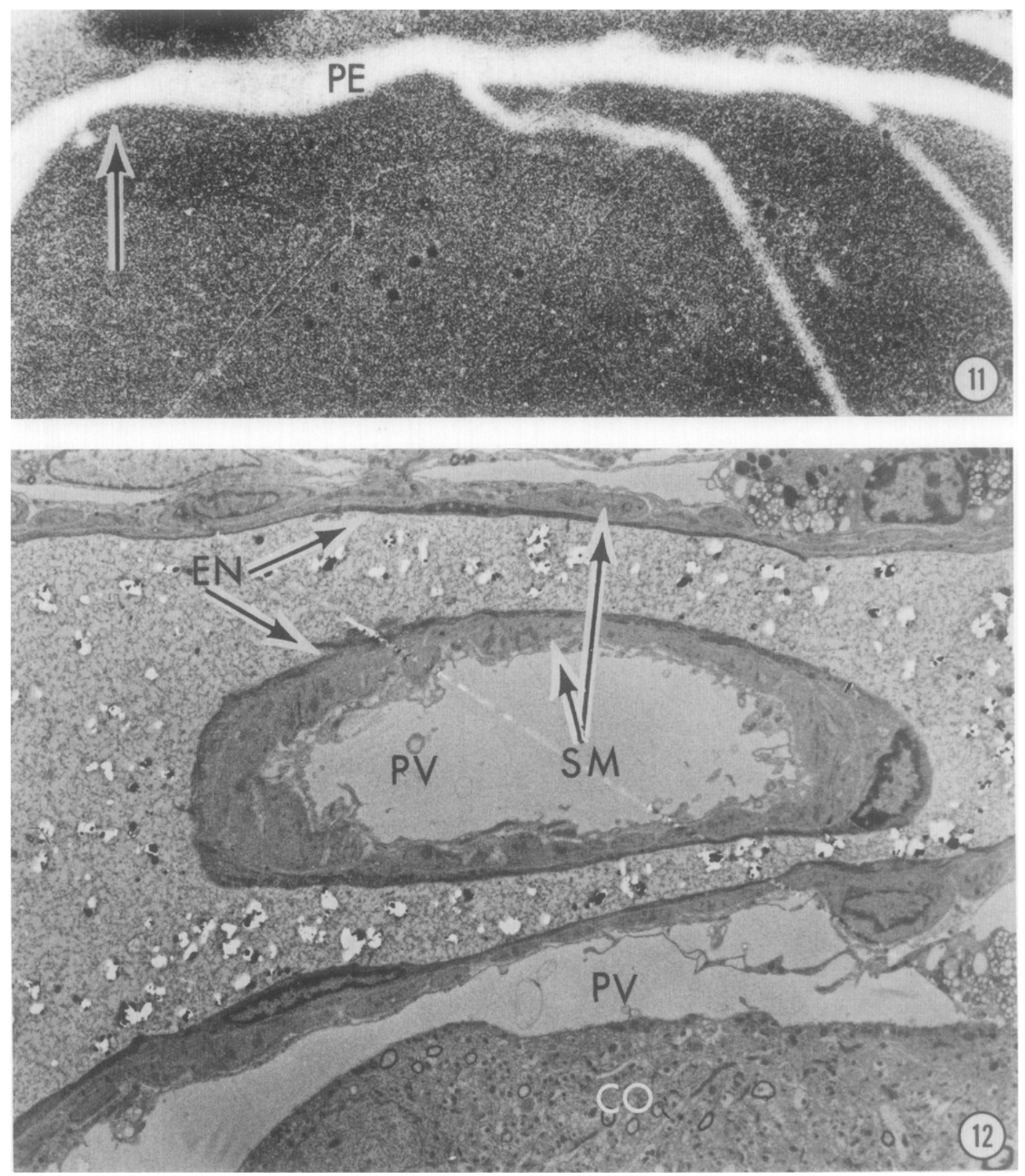

FIG. 11. Microangiograph of a cortical penetrating arteriole (PE) from a stroke-prone rat, showing a constriction in the vessel image (arrow). $\times 200$.

FIG. 12. Electron micrograph of the arteriole in Fig. 9, showing perivascular edema (PV) in association with invagination of the vascular wall. Note the inversion of medial (SM) and endothelial (EN) components at the site of invagination. $\mathrm{CO}$, cortical parenchyma. $\times 2200$.

\section{Fixative Osmolarities}

Hyperosmolar fixatives, although providing optimal neural preservation could be expected to cause difficulties in studies of the vascular system because of its sensitivity to osmotic changes. Hyperosmolarity has been reported to increase vascular permeability, possibly through opening of endothelial cell junctions $(15,16,18)$. Whereas, total osmolarities of our fixatives (as indicated by freezing-point depression) are high because of the aldehyde contribu- 
tion, aldehydes are generally considered to contribute little to the actual effective osmolarity $(1,9,10)$. If so, our weak fixative, at least, would be approximately isotonic with blood plasma values.

\section{Weak and Strong Fixatives}

The two-step perfusion method, introduced by Brightman and Reese (2) in the present study yielded optimal preservation of both neural parenchyma and vascular components. The weak fixative presumably provided primary fixation without inducing changes seen in the vascular wall with concentrated fixative alone. However, the subsequent introduction of the more concentrated fixative appeared to further stabilize deepest parenchymal components $(1,5)$ and resulted in better preservation than when weak fixative was used alone.

\section{Perfusion Pressures}

To avoid pressure-induced changes to the vascular endothelium and a resultant disruption of the blood-brain barrier, it was necessary to perfuse at pressures within the physiological range. Perivascular or glial edema were observed in normal animals only when perfused at pressures in excess of $100 \mathrm{~mm} \mathrm{Hg}$. These high-perfusion pressures resulted in structural changes such as flattening of endothelial pinocytotic vesicles, presumably due to forced stretching of the vessel wall. Approximately constant pressures (within 10\%) were maintained by the gravity driven apparatus, allowing the flow rate to accommodate to any changes in resistance during the change from resilient to fixed condition of the vessels.

The addition of Evan's blue marker to flush and fixative solutions demonstrated no vascular permeability changes resulting from either solution osmolarities or perfusion pressures.

\section{Preparation for Microangiography}

A problem common in microangiographic studies has been the uneven vas- cular filling achieved by perfusion with radiographic contrast material. Preheparinization and vascular flush prevented the blood cell aggregation and the interaction of fixative with blood plasma components which were observed in nontreated animals as occlusions to the free movement of contrast material through the vasculature.

\section{Contrast Medium Viscosity and Perfusion Pressures}

Elevated perfusion pressure has commonly been regarded as the primary means of effecting optimal filling $(3,6)$. In contrast, the necessity in the present study of avoiding pressure effects on vascular ultrastructure and of limiting filling to the arterial side of the vascular bed required the use of relatively low perfusion pressures (120-130 $\mathrm{mm} \mathrm{Hg}$ ) and correspondingly reduced viscosity of the contrast material. However, in order to retain the radiographic material within vessels during manipulating and slicing of tissue for microangiography and subsequent electron microscopy, it was necessary to provide for the congealing of the radiographic medium by the addition of gelatin (17). The preinfusion flush emptied the vasculature of aldehydes, allowing the gelatin base medium to be freely infused. Its gelatin was effected by the subsequent rediffusion of aldehydes from parenchyma to the vessels. A concentration of $2 \%$ gelatin in a $25 \%$ aqueous dilution of Micropaque provided adequate but not premature congelation of the medium while sufficiently lowering viscosity without compromising radiological opacity. Density of barium sulfate granules in thin sections was sufficient to serve as electron microscopic markers (Figs. 5, 8, and 10). The contrast medium was infused at $40-42^{\circ} \mathrm{C}$ to keep the gelatin fluid. A systematic study of various parameters associated with microangiographic filling has been performed, but predominantly with respect to unfixed brain tissues (11). 


\section{Selective Arteriolar Filling}

A persistent problem in microvascular studies has been the need for a dependable means of distinguishing arteriolar from venular segments. The modified radiographic contrast material, when perfused via aorta at $120-130 \mathrm{~mm} \mathrm{Hg}$ provided an excellent marker for this purpose. The obstruction of contrast material by the capillaries may result from sedimentation or packing of the barium sulfate granules at the reduced pressures and flow rates experienced at the capillary level. Alternately, the use of higher perfusion pressures $(150-200 \mathrm{~mm}$ $\mathrm{Hg}$ ) alone or in conjunction with extended perfusion times resulted in nonselective vascular filling similar to that generally observed in angiographic studies.

\section{General Applicability}

By means of the combined methodology it was possible to rapidly scan entire rat cerebral arterial systems, to determine patterns in the distribution of features of interest (luminal constrictions, in the present study), and to efficiently isolate and analyze those features as to their differing structural and ultrastructural basis. While this methodology was developed primarily with respect to cerebral vascular studies, it has been successfully applied to the vasculature of retina, kidney, and thoracic musculature. Our results have indicated its effectiveness for systematic ultrastructural analysis of features down to the smallest microvascular level. Orientation with respect to the whole organ or vascular network is preserved, and because the same feature is observed by the two techniques, it permits a correlation between gross and ultrastructural observations not otherwise attainable.

This work was supported by National Institute of
Health Research Grants HL-18575-03 and HL-1883103. The authors wish to thank Mrs. Gunilla Jokelainen for her invaluable technical assistance and Dr. Theodore O. Sippel for his help with the manuscript.

\section{REFERENCES}

1. Arborgh, B., Belu, P., Brunk, U., And Collins, V. P. (1976) J. Ultrastruct. Res 56, 339-350.

2. Brightman, M. W., And Reese, T. S. (1969) $J$. Cell Biol. 40, 618-676.

3. Clark, R., Mandel, S., and Webster, W. (1977) Invest. Radiol. 12, 62-73.

4. Fernando, D. A., and Lad, J. K. H. (1978) Acta Anat. 100, 241-249.

5. Forssmann, W. G., Ito, S., Weine, E., Aoki, A., Dym, M., And FAwcett, D. W. (1977) Anat. Rec. 188, 307-314.

6. GADE, R. (1977) Invest. Radiol. 12, 348-352.

7. Garcia, J. H., Kalimo, H., Kamiryo, Y., and Trump, B. F. (1977) Virchows Arch. B 25, 191206.

8. Gremminger, R. F., Baden, J. B., Sellers, R. D., et al. (1970) Invest. Radiol. 5, 275-276.

9. Kalimo, H., Garcia, J. H., KamiJko, Y., Tanaka, J. AND Trump, B. F. (1977) Virchows Arch. $B$ 25, 207-220.

10. Karlson, U., ANd SChultz, R. C. (1965) I. Ultrastruct. Res. 12, 160-186.

11. Kormano, M., and Reidonen, K. (1973) Neuroradiology 5, 83-86.

12. Metz, J., Aoki, A., and Forssmann, W. G. (1978) Cell Tissue Res. 192, 319-407.

13. OKamoto, K., Yamori, Y., and Napaoka, A. (1974) Circ. Res, 34-35 (Suppl. 1), 143-153.

14. Peters, A. (1970) in Nauta, W. J., And EbbesSON, S. O. (Eds.), Contemporary Research Methods in Neuroanatomy, pp. 56-76, SpringerVerlag, New York.

15. Rapoport, S. I., Matthew, K., Thomson, H. K., and Pettigrew, K. D. (1977) Brain Res. 136, $23-29$.

16. RAPOPORT, S. I. (1973) in HILAL, S. K., et al. (Eds.), Small Vessell Angiography, pp. 137-151, Mosby, St. Louis, Mo.

17. SChlesingER, M. J. (1957) Lab Invest. 6, 1-11.

18. Sterrett, P. R., Thompson, A. M., Chapmen, A. L., AND Matzke, H. A. (1974) Brain Res. 77, 281-295.

19. Williams, T. H., and Jew, J. Y. (1975) Tissue Cell 7, 407-418. 